\title{
Iniciação Científica Entomofauna capturada em pomar recém-implantado de pessegueiro e ameixeira no município de Buri, São Paulo, Brasil
}

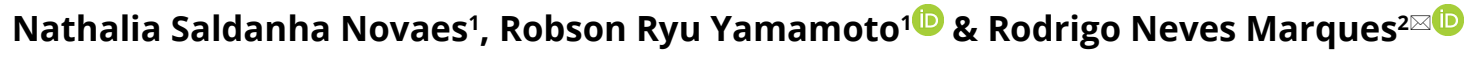

1. Centro de Ciências da Natureza, Universidade Federal de São Carlos - UFSCar, Buri-SP, Brasil. 2. Departamento de Biotecnologia e Produção Vegetal e Animal, Centro de Ciências Agrárias, Universidade Federal de São Carlos - UFSCar, Araras-SP, Brasil.

\section{Entomology Beginners, vol. 2: e021 (2021)}

Resumo. O cultivo de frutíferas de caroço está entre as atividades agrícolas de maior importância na fruticultura brasileira, com destaque para o pessegueiro [Prunus persica (L.) Batsch] e a ameixeira (Prunus domestica L.). Insetos-pragas constituem uma grande ameaça para a fruticultura nacional e, para seu manejo eficiente e racional, é primordial o conhecimento quali-quantitivo de insetos potenciais pragas e inimigos naturais. Este trabalho teve como objetivo realizar o levantamento e análise faunística de insetos ocorrentes em pomar de pessegueiro $(P$. persica vr. Kampai) e ameixeira (P. domestica vr. Reubennel) no município de Buri, São Paulo. Foram utilizados três tipos de armadilhas: caça-mosca, cartões adesivos amarelos e Moericke (com água e sabão). As coletas foram realizadas quinzenalmente de setembro de 2018 a março de 2019 . Verificouse grande diversidade e abundância de insetos na área estudada, sendo as famílias Aphidae, Formicidae, Chrysomelidae, Dolichopodidae, Muscidae, Otitidae as mais frequentes. A análise faunística realizada nesta pesquisa mostrou que houve incidência de potenciais pragas secundárias, membros das famílias Drosophilidae, Membracidae, Aphidae, Formicidae e Chrysomelidae. Também foi observada diversidade de inimigos naturais das famílias Spongiphoridae, Vespidae, Ichneumonidae e Coccinellidae. A adoção de medidas conservacionistas de inimigos naturais é fundamental para evitar surtos populacionais de potenciais pragas em áreas produtoras.

Palavras-chave: Inimigos naturais; Ecologia de insetos; Manejo integrado de pragas.

\section{Entomofauna captured in a newly planted peach and plum orchard in the municipality of Buri, São Paulo state, Brazil}

Abstract. Stone fruits are considered one of the most important agricultural activities in Brazilian fruit crops, especially peach [Prunus persica (L.) Batsch] and plum (Prunus domestica L.). Insect pests are a huge threat to the Brazilian fruticulture and, to its rational and efficient management, is important to know the quali-quantitative insects pests and natural enemies. The aim of this work was to carry out survey and faunistic analysis of insects with occurrence in peach ( $P$. persica vr. Kampai) and plum $(P$. domestica vr. Reubennel) in the municipality of Buri, São Paulo state, Brazil. We used three types of traps: fruit fly trap, yellow adhesive cards and Moericke traps (water + soap). The surveys were performed from September 2018 to March 2019, with biweekly frequency. The survey pointed a high diversity and abundance of insects in the studied area, being the families Aphidae, Formicidae, Chrysomelidae, Dolichopodidae, Muscidae and Otitidae with high frequency. The faunistic analysis showed a high incidence of potential secondary pests, composed by the families Drosophilidae, Membracidae, Aphidae, Formicidae, and Chrysomelidae. In addition, it was observed diversity of natural enemies of the families Spongiphoridae, Vespidae, Ichneumonidae and Coccinellidae. Adoption of conservationist management to preserve natural enemies is essential to avoid outbreaks of potential pests.

Keywords: Natural enemies; Insect ecology; Integrated pest management.

O cultivo de frutíferas de caroço está entre as atividades agrícolas de maior importância na fruticultura brasileira, com destaque para o pessegueiro, Prunus persica (L.) Batsch, e para a ameixeira, Prunus domestica L. (Rosales: Rosaceae) (ANUÁRIO..., 2020). Contudo, os insetos-praga constituem uma ameaça e desafio aos produtores, principalmente por se tratarem de inúmeras espécies, esporádicas ou constantes, que causam perdas econômicas significativas. Além dessas perdas diretas, também provocam prejuízos ambientais, principalmente em detrimento ao uso contínuo de inseticidas (COELHO et al., 2008) e dificultam a exportação de frutos, devido às restrições quarentenárias impostas pelos países importadores
(LOHMANN, 2018).

Programas de Manejo Integrado de Pragas (MIP) em fruticultura têm incentivado o uso de diferentes métodos e táticas de controle, contribuindo com a diminuição dos impactos ambientais e favorecendo o aumento da população de organismos benéficos (TIBOLA et al., 2005; NAKANO, 2011; ARIOLI et al., 2016). A amostragem de populações de insetos é uma das bases do MIP, sendo primordial para que as várias táticas de controle de pragas possam ser utilizadas com critério e em tempo adequado. A análise faunística, aplicada em estudos ecológicos, é um recurso utilizado para determinar as

\section{Editado por:}

Rodrigo Souza Santos

\section{Histórico Editoral:}

Recebido em: 28.07.2021

Aceito em: 28.09.2021

Publicado em: 03.11.2021

\section{Autor Correspondente:}

Rodrigo Neves Marques

rodrigo.marques@ufscar.br

Agência(s) de Fomentos:

Conselho Nacional de Desenvolvimento Científico e Tecnológico - CNPq 
espécies predominantes em agroecossistemas e quantificar os índices ecológicos de grupos taxonômicos, gerando informação para aplicação em programas de Manejo Ecológico de Pragas (SILVEIRA NETO et al., 1976; RAMOS et al., 2016).

Para o estabelecimento de programas de controle de pragas, torna-se necessário o prévio conhecimento dos aspectos ecológicos das populações, a exemplo da diversidade de espécies, frequência, dominância, constância, entre outros parâmetros faunísticos. Esses são parâmetros fundamentais no estabelecimento de boas estratégias de manejo integrado. Desta forma, para a implementação do MIP, é de extrema importância o levantamento dos grupos taxonômicos presentes na área para toda a cadeia de ações a serem tomadas ao longo do cultivo (GALLO et al., 2002).

A análise faunística e o conhecimento prévio sobre a entomofauna (benéfica ou não) ocorrente em pomares, são essenciais no desenvolvimento de estratégias para a adoção de práticas de manejo conservacionistas (SILVEIRA NETO et al., 1976). Assim, o presente trabalho teve como objetivo realizar o levantamento de insetos ocorrentes em um pomar recémimplantado de pessegueiro e ameixeira no estado de São Paulo.

O trabalho foi conduzido em área de pomar didáticoexperimental de pessegueiro (variedade Kampai) e ameixeira (variedade Reubennel), com aproximadamente 200 plantas de cada espécie, dispostas de forma concentrada, com espaçamento entre plantas de $3 \times 6 \mathrm{~m}$, em uma área total de 1

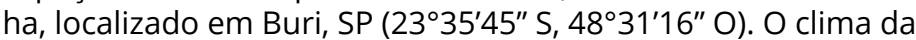
região é do tipo subtropical úmido - classificação climática de Köppen-Geiger: Cfa (MARTINELLI, 2010).

As plantas apresentavam 1,5 ano pós-transplantio e aproximadamente $2 \mathrm{~m}$ de altura. A área avaliada faz divisa a um pequeno fragmento de mata preservada, uma lagoa e áreas comerciais de produção de grãos (soja verão, milho safrinha e trigo de inverno), com cerca de $50 \mathrm{~m}$ de distância de tais localidades. Antes da instalação, a área era cultivada com grãos, sem histórico de frutíferas na área. Durante o período de coleta não foram realizadas aplicações de agrotóxicos na área do estudo, porém as áreas adjacentes receberam aplicações frequentes de agrotóxicos (inseticidas, herbicidas e fungicidas) e fertilizantes.

A área do estudo foi monitorada de setembro de 2018 a março de 2019, com a realização de coletas quinzenais, totalizando 15 coletas. Foram utilizados três tipos diferentes de armadilhas: i) armadilha caça-mosca (confeccionada com garrafas PET de $2 \mathrm{~L}$ ), contendo proteína hidrolisada (Dismel ${ }^{\circledR}$ ), à $5 \%$ de concentração; ii) cartão adesivo amarelo $(30 \times 10 \mathrm{~cm})$ e; iii) armadilhas de Moericke (bandejas amarelas de $19 \times 19 \times 8,5$ $\mathrm{cm})$, contendo água e sabão detergente $(0,5 \%)$ (Figuras $1 \mathrm{~A}, \mathrm{~B}$ e C).
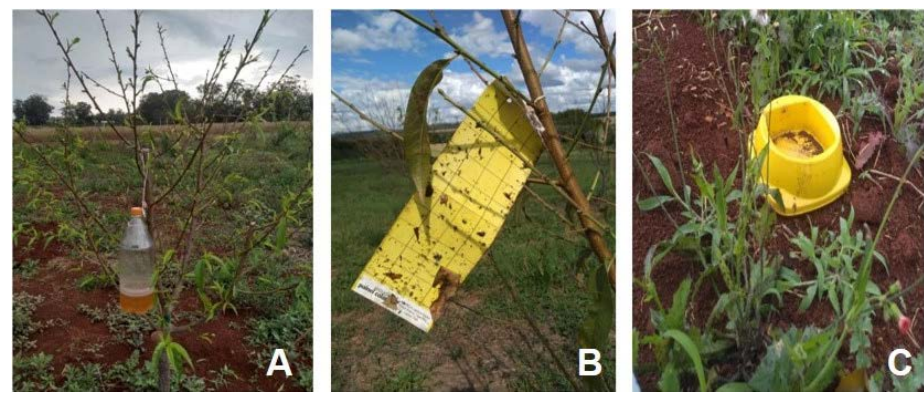

Figura 1. Armadilhas utilizadas para a captura de insetos em área de plantio de pêssego e ameixa, Buri, SP. A. Armadilha tipo caça-mosca confeccionada com garrafa PET de $2 \mathrm{~L}$, contendo proteína hidrolisada (5\%); B. cartão adesivo amarelo $(30 \times 10 \mathrm{~cm}) ;$ C. armadilha de Moericke (bandejas amarelas de $19 \times 19 \times 8,5 \mathrm{~cm}$ ), com água e sabão detergente $(0,5 \%)$.

As armadilhas foram instaladas aleatoriamente na área do pomar, distribuídas de forma a abranger toda a área monitorada, com distância mínima de 20 m entre elas. Ao todo, foram instaladas seis armadilhas de bandejas amarelas com água, nove cartões adesivos e quatro armadilhas caçamoscas. As armadilhas caça-moscas e os cartões adesivos foram fixados em plantas a $1 \mathrm{~m}$ de altura do solo (tanto em ameixeira quanto em pessegueiro), já as bandejas amarelas foram alocadas nas entrelinhas. Quinzenalmente, a proteína hidrolisada das armadilhas caça-moscas era substituída e as bandejas amarelas eram reabastecidas com água e sabão. Os cartões adesivos foram substituídos a cada 30 dias.

O material de cada coleta e armadilha foi armazenado em frascos individualizados de vidro contendo etanol a 70\%, para posterior avaliação. Em laboratório, os espécimes foram triados, contabilizados e identificados, em nível de ordem e família. Para a identificação dos espécimes, foi utilizado um microscópio estereoscópico binocular, seguindo as chaves dicotômicas para insetos comumente encontrados em ambientes agrícolas (GALLO et al., 2002; FUJIHARA et al., 2016).

Os dados obtidos foram submetidos à análise faunística por meio do software ANAFAU (MORAES et al., 2003), sendo obtidos os índices de dominância (dominante e não dominante), abundância (rara, dispersa, comum, abundante e muito abundante), frequência (muito frequente, frequente e pouco frequente) e constância (constante, acessória e acidental).

Foi coletado um total de 11.056 indivíduos, pertencentes a nove ordens e 36 famílias. As famílias consideradas predominantes foram aquelas que se destacaram por obter os maiores índices faunísticos de abundância, frequência, constância e dominância (Tabela 1).

As famílias Aphidae (Hemiptera), Formicidae (Hymenoptera), Chrysomelidae (Coleoptera), Dolichopodidae, Muscidae e Otitidae (Diptera) foram mais frequentes, seguidas por Membracidae, Cicadellidae (Hemiptera), Apidae, Vespidae (Hymenoptera) e Drosophilidae (Diptera) (Tabela 1).

Não foram encontrados espécimes pragas-chave das culturas neste estudo, como por exemplo, indivíduos da família Tephritidae (Diptera) e Tortricidae (Lepidoptera) (ZART et al., 2011). A ausência destes insetos pode estar atrelada ao fato de a área de estudo não possuir histórico de cultivo de plantas hospedeiras (neste caso, de espécies frutíferas). Além disso, a elevada diversidade de insetos na área estudada pode estar associada à uma maior complexidade de interações ecológicas, impedindo a ocorrência de insetos pragas, os quais apresentam maior adaptabilidade em áreas com desequilíbrio biológico (ALTIERI et al., 2003). Áreas com fragmentos de mata adjacentes podem exercer efeito quali-quantitativo sobre a presença de inimigos naturais em áreas agrícolas (BORTOLOTTO et al., 2016; MILANI et al., 2020).

No entanto, a análise faunística realizada mostrou que há alta incidência de potenciais pragas secundárias, principalmente das famílias Membracidae, Aphidae, Cicadellidae (Hemiptera), Thripidae (Thysanoptera), Drosophilidae (Diptera), Formicidae (Hymenoptera) e Chrysomelidae (Coleoptera).

O levantamento também apontou para uma grande diversidade e abundância de outros insetos na área estudada, com destaque para potenciais inimigos naturais, como Spongiphoridae, Vespidae, Ichneumonidae e Coccinellidae. Representantes destas famílias em cultivos agrícolas participam ativamente do controle de insetos (PARRA et al., 2002), sendo responsáveis por evitar a ocorrência de surtos populacionais de insetos, que podem atingir o status de praga (NAKANO, 2011). A família Formicidae é um exemplo da presença tanto de potenciais pragas (Atta spp., Acromyrmex spp.), quanto de inimigos naturais (Solenopsis spp.) (GALLO et al., 2002; PARRA et al., 2002; SUJII et al., 2020).

Apesar de serem classificados como raros ou pouco frequentes, indivíduos da ordem Dermaptera também foram encontrados na área de estudo. A presença de insetos desta ordem em 
plantações de pessegueiros é de extrema importância para a manutenção de insetos abaixo do nível de dano econômico, principalmente em períodos de dormência desta espécie de frutífera (POLTRONIERI et al., 2008).

A análise faunística é um bom indicativo da potencial presença de pragas e inimigos naturais nos sistemas (SILVEIRA NETO et al., 1976, GIUSTOLIN et al., 2009). No entanto, quando há famílias de insetos com representantes de diferentes hábitos alimentares, torna-se complexa a determinação do efeito positivo ou negativo de determinada família. Para uma compreensão mais aprofundada, seria fundamental a classificação em nível de espécie, o que não foi o objetivo do presente trabalho.

A alta diversidade de famílias e ordens de insetos pode estar relacionada à influência das áreas no entorno do pomar, principalmente ao fragmento de mata preservada (SILVEIRA NETO et al., 1995; SARTOR et al., 2010; BORTOLOTTO et al., 2016; MILANI et al., 2020), que se encontrava cerca de $50 \mathrm{~m}$ de distância da área de estudo. Associado a isso, o isolamento da área quanto à aplicação de agrotóxicos pode ter contribuído para maior diversidade de famílias (SOUZA et al., 2018). Essa condição pode favorecer a disponibilidade de alimento, beneficiando o estabelecimento e desenvolvimento de insetos com diferentes hábitos alimentares na área estudada (BERNARDI et al., 2013; SOUZA et al., 2018). A presença de áreas adjacentes também exerce influência em áreas de cultivos de cafeeiro e de laranja-doce, onde a influência deste tipo de vegetação garante elevado índice de similaridade diferentes paisagens agrícolas quanto à diversidade de espécies (GIUSTOLIN et al., 2009).

Em cultivos de pessegueiro ocorrem várias espécies de

Tabela 1. Índices faunísticos, ordens, famílias e número de indivíduos coletados em pomar de pessegueiro e ameixeira no município de Buri, SP no período de setembro de 2018 a março de 2019.

\begin{tabular}{|c|c|c|c|c|c|c|c|}
\hline \multirow{2}{*}{ Ordem } & \multirow{2}{*}{ Família } & \multirow{2}{*}{$N^{\circ}$ de indivíduos } & \multirow{2}{*}{ Coletas com ocorrência do táxon } & \multicolumn{4}{|c|}{ Índices faunísticos* } \\
\hline & & & & D & A & $\mathbf{F}$ & C \\
\hline \multirow{12}{*}{ Hemiptera } & Membracidae & 322 & 15 & $\mathrm{D}$ & c & $\mathrm{F}$ & w \\
\hline & Cicadellidae & 389 & 15 & D & c & $\mathrm{F}$ & w \\
\hline & Aphidae & 1.253 & 15 & $\mathrm{D}$ & ma & MF & w \\
\hline & Pentatomidae & 4 & 4 & ND & r & $\mathrm{PF}$ & $\mathrm{y}$ \\
\hline & Coreidae & 12 & 12 & $\mathrm{D}$ & r & $\mathrm{PF}$ & w \\
\hline & Cercopidae & 2 & 2 & ND & r & $\mathrm{PF}$ & z \\
\hline & Rhopalidae & 44 & 15 & $\mathrm{D}$ & $r$ & PF & w \\
\hline & Miridae & 32 & 15 & D & $r$ & $\mathrm{PF}$ & w \\
\hline & Reduvidae & 25 & 15 & D & $r$ & PF & w \\
\hline & Alydidae & 1 & 1 & ND & $r$ & $\mathrm{PF}$ & z \\
\hline & Pyrrhocoridae & 3 & 3 & ND & $r$ & PF & z \\
\hline & Largidae & 4 & 4 & ND & $r$ & $\mathrm{PF}$ & $y$ \\
\hline \multirow{5}{*}{ Hymenoptera } & Ichneumonidae & 4 & 4 & ND & $r$ & PF & $\mathrm{y}$ \\
\hline & Apidae & 189 & 15 & D & c & $\mathrm{F}$ & w \\
\hline & Vespidae & 196 & 15 & D & c & $\mathrm{F}$ & w \\
\hline & Formicidae & 1.220 & 15 & D & ma & MF & w \\
\hline & Sphecidae & 23 & 15 & D & $r$ & PF & w \\
\hline \multirow{2}{*}{ Dermaptera } & Spongiphoridae & 21 & 15 & D & $r$ & $\mathrm{PF}$ & w \\
\hline & Labiduridae & 4 & 4 & ND & $r$ & $\mathrm{PF}$ & $\mathrm{y}$ \\
\hline \multirow{4}{*}{ Orthoptera } & Acrididae & 33 & 15 & $\mathrm{D}$ & $r$ & PF & w \\
\hline & Gryllidae & 5 & 5 & ND & $r$ & $\mathrm{PF}$ & $\mathrm{y}$ \\
\hline & Ommexechidae & 1 & 1 & ND & $r$ & PF & z \\
\hline & Romaleidae & 1 & 1 & ND & $r$ & PF & z \\
\hline Neuroptera & Chrysopidae & 9 & 9 & D & $r$ & $\mathrm{PF}$ & w \\
\hline \multirow{5}{*}{ Coleoptera } & Staphylinidae & 44 & 15 & D & $r$ & PF & w \\
\hline & Brentidae & 1 & 1 & ND & $r$ & $\mathrm{PF}$ & w \\
\hline & Cerambycidae & 12 & 12 & D & $r$ & PF & w \\
\hline & Chrysomelidae & 1.370 & 15 & D & ma & MF & w \\
\hline & Coccinelidae & 97 & 15 & $\mathrm{D}$ & $d$ & $\mathrm{PF}$ & w \\
\hline \multirow{5}{*}{ Diptera } & Dolichopodidae & 1.760 & 15 & D & ma & MF & w \\
\hline & Stratiomyidae & 10 & 10 & D & $r$ & $\mathrm{PF}$ & w \\
\hline & Muscidae & 1.840 & 15 & D & ma & MF & w \\
\hline & Otitidae & 1.350 & 15 & $\mathrm{D}$ & ma & MF & w \\
\hline & Drosophilidae & 220 & 15 & D & c & $\mathrm{F}$ & w \\
\hline Thysanoptera & Thripidae & 520 & 15 & D & $\mathrm{a}$ & MF & w \\
\hline Lepidoptera & Noctuidae & 35 & 15 & $\mathrm{D}$ & $r$ & $\mathrm{PF}$ & w \\
\hline
\end{tabular}

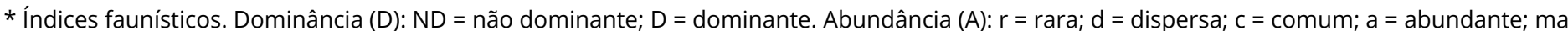
= muito abundante. Frequência $(F): M F=$ muito frequente; F: frequente; $P F=$ pouco frequente. Constância $(C): w=$ constante; $y=a c e s s o ́ r i a ; ~ z=$ acidental (Silveira Neto et al., 1976). 
inimigos naturais, sendo a maior parte de representantes das ordens Hymenoptera e Coleoptera (SCHUBER et al., 2012). O presente estudo demonstra que essas ordens foram altamente representativas, com potenciais inimigos naturais pertencentes às famílias Vespidae, Formicidae, Ichneumonidae (Hymenoptera), Staphilinidae e Coccinelidae (Coleoptera). Tais informações podem ser utilizadas para o estabelecimento e uso de estratégias de manejo conservativo de inimigos naturais, o que resulta um maior equilíbrio nas áreas de cultivo (FONTES et al., 2020).

Análises faunísticas realizadas em cultivo de Eucalyptus spp. realizadas por Bernardi et al. (2011) também demonstraram predominância de determinadas famílias. Este fato pode estar associado à disponibilidade de alimento favorável ao estabelecimento e desenvolvimento de insetos na área de estudo.

Os resultados obtidos salientam a importância da realização das práticas conservacionistas de manejo de pragas (ALTIERI et al., 2003; FONTES et al., 2020). O MIP se baseia no monitoramento da praga e de seus inimigos naturais, e qualquer tomada de decisão deve ser fundamentada pelo conhecimento do nível populacional e grau de severidade da praga em relação à cultura, bem como do potencial de controle dos organismos que regulam as populações dessas pragas (GALLO et al., 2002; O'NEIL e OBRYCKI, 2008).

O controle racional e eficiente dos insetos praga tem como pré-requisito o conhecimento do momento adequado para a adoção de medidas de controle (NAKANO, 2011). Desta forma, o conhecimento sobre a flutuação populacional dos insetos incidentes no local, inimigos naturais, bem como as condições climáticas, tornam-se fatores indispensáveis para um manejo adequado dos cultivos agrícolas (GRAY et al., 2008).

O pomar misto de pessegueiro e ameixeira, sem histórico de cultivo comercial destas espécies frutíferas no local estudado, não apresenta indivíduos pragas chave. Contudo, a análise faunística indica abundância de potenciais pragas secundárias e inimigos naturais.

\section{AGRADECIMENTOS}

Ao Conselho Nacional de Desenvolvimento Científico e Tecnológico - CNPq, pelo financiamento da bolsa de iniciação científica da primeira autora (processo n² 160903/2018-1).

\section{REFERÊNCIAS}

ALTIERI, M. A.; SILVA, E. N.; NICHOLLS, C. I. (Eds.). O papel da biodiversidade no manejo de pragas. Ribeirão Preto: Holos, 2003. 225p.

ANUÁRIO BRASILEIRO DE HORTI \& FRUTI. Produção brasileira de frutas permanece estabilizada, com o volume estimado em 43 milhões de toneladas em 2019, beneficiada pelo clima. Santa Cruz do Sul, RS. 2020. Disponível em: <http://www. editoragazeta.com.br/sitewp/wp-content/uploads/2020/05/ HORTIFRUTI_2020.pdf>. Acesso em: 30 set. 2021.

ARIOLI, C. J.; BOTTON, M.; AZEVEDO-FILHO, W. S.; MENEZESNETTO, A. C.; ROSA, J. M. Manejo dos principais insetos e ácaros-praga na cultura da ameixeira no Sul do Brasil. Florianópolis: Epagri (Boletim técnico, 174), 2016. 46p.

BERNARDI, D.; ARAUJO, E. S.; ZAWADNEAK, M. A. C.; BOTTON, M.; MOGOR, A. F.; GARCIA, M. S. Aphid species and population dynamics associated with strawberry. Neotropical Entomology, v. 42, p. 628-633, 2013. DOI: https://doi.org/10.1007/s13744-

\section{3-0153-1}

BERNARDI, O.; GARCIA, M. S.; SILVA, E. J. E.; ZAZYCKI, L. C. F.; BERNARDI, D.; FINKENAUER, E. Levantamento populacional e análise faunística de Lepidoptera em Eucalyptus spp. no município de Pinheiro Machado, RS. Ciência Florestal, v. 21, n. 4, p. 735-744, 2011. DOI: https://doi.org/10.5902/198050984517

BORTOLOTTO, O. C.; MENEZES JUNIOR; A. O.; HOSHINO, A. T. Abundância de inimigos naturais de pulgões do trigo em diferentes distâncias da borda da mata. Pesquisa Agropecuária Brasileira, v. 51, n. 2, p. 187-191, 2016. DOI: https://doi.org/10.1590/S0100-204X2016000200011

COELHO, L. R.; LEONEL, S.; CROCOMO, B. W.; LABINAS, A. M. Controle de pragas no pessegueiro através do ensacamento de frutos. Ciência e Agrotecnologia, v. 32, n. 6, p. 1743-1747, 2008. DOI: https://doi.org/10.1590/S1413-70542008000600010

FONTES, E. M. G; PIRES, C. S. S.; SUJII, E. R. Estratégias de uso e histórico. pp. 21-40. In: FONTES, E. M. G. VALADARES-INGLIS, M. C. (Eds.). Controle biológico de pragas da agricultura. Brasília: Embrapa, 2020. 510p.

FUJIHARA, R. T.; FORTI, L. C.; ALMEIDA, M. C.; BALDIN, E. L. L. (Eds.). Insetos de importância econômica: guia ilustrado para identificação de famílias. Botucatu: FEPAF, 2016. 391 p.

GALLO, D.; NAKANO, O.; SILVEIRA NETO, S.; CARVALHO, R. P. L.; BAPTISTA, G. C.; BERTI FILHO, E.; PARRA, J. R. P.; ZUCCHI, R. A.; ALVES, S. B.; VENDRAMIN, J. D.; MARCHINI, L. C.; LOPES, J. R. S.; OMOTO, C. (Eds.). Entomologia agrícola. Piracicaba: FEALQ, 2002. 920p.

GIUSTOLIN, T. A.; LOPES, J. R. S.; QUERINO, R. B.; CAVICHIOLI, R. R.; ZANOL, K.; AZEVEDO FILHO, W. S.; MENDES, M. A. Diversidade de Hemiptera Auchenorrhyncha em Citros, Café e Fragmento de Floresta Nativa do Estado de São Paulo. Neotropical Entomology, v. 38, n. 6, p. 834-841, 2009.

GRAY, M.; RATCLIFFE, S.; RICE, M. The IPM paradigm: Concepts, strategies and tactics. pp.1-13. In: RADCLIFFE, E.; HUTCHISON, W.; CANCELADO, R. (Eds.). Integrated Pest Management: concepts, tactics, strategies and case studies. Cambridge: Cambridge University Press, 2008. 529p. DOI: https://doi. org/10.1017/CBO9780511626463.002

LOHMANN, T. R. Panorama da priorização de pragas no mundo. pp. 23-28. In: FIDELIS, E. G.; LOHMANN, T. R.; SILVA, M. L. da; PARIZZI, P.; BARBOSA, F. F. L. (Eds.). Priorização de pragas quarentenárias ausentes no Brasil. Brasília: Embrapa, 2018. 510p.

MARTINELLI, M. Clima do Estado de São Paulo. Confins. 2010. Disponível em <http://journals.openedition.org/confins/6348>. Acesso em: 30 set. 2021.

MILANI, L. R.; JACQUES, G. C.; CLEMENT, M. A.; COELHO, E. L.; SOUZA, M. M. Influência de fragmentos florestais sobre a nidificação de vespas sociais (Hymenoptera, Vespidae) em cafeeiro. Revista Brasileira de Zoociências, v. 21, n. 1, p. 1-12, 2020.

MORAES, R. C. B.; HADDAD, M. L.; SILVEIRA NETO, S.; REYES, A. E. 
L. Software para análise faunística. In: SIMPÓSIO DE CONTROLE BIOLÓGICO - SICONBIOL, 8. Anais... São Pedro, SP: ESALQ, 2003. p. 195.

NAKANO, O. (Ed.) Entomologia econômica. $2^{\text {a }}$ ed. Piracicaba: O Autor, 2011. 464p.

O'NEIL, R.; OBRYCKI, J. Introduction and augmentation of biological control agents. pp. 105-117. In: RADCLIFFE, E.; HUTCHISON, W.; CANCELADO, R. (Eds.). Integrated Pest Management: concepts, tactics, strategies and case studies. Cambridge: Cambridge University Press, 2008. 529p. DOI: https://doi.org/10.1017/CBO9780511626463.010

PARRA, J. R. P.; BOTELHO, P. S. M.; CORRÊA-FERREIRA, B. S.; BENTO, J. M. S. (Eds.). Controle biológico no Brasil: parasitóides e predadores. São Paulo: Manole, 2002. 635p.

POLTRONIERI, A. S.; SCHUBER, J. M.; ZAWADNEAK, M. A. C.; CARDOSO, N. A. Ocorrência e flutuação populacional de tesourinhas em pomares de pessegueiro no município de Araucária, Paraná. Scientia Agraria, v. 9, n. 4, p. 551-554, 2008. DOI: http://dx.doi.org/10.5380/rsa.v9i4.12511

RAMOS, M. A.; DA SILVA, H. C. H.; ALVES, A. G. C. Análise faunística de insetos associados à cultura do quiabeiro [Abelmoschus esculentus (L.) Moench] em plantio comercial, no município de Canindé de São Francisco, CE, Brasil. EntomoBrasilis, v. 9, n. 2, p. 146-149, 2016. DOI: https://doi.org/10.12741/ebrasilis. v9i2.605

SARTOR, V.; WOLDAN, D. R. H.; GARCIA, F. R. M. Inventário e aspectos ecológicos da fauna entomológica no município de União da Vitória Paraná. Biodiversidade Pampeana, v. 7, n. 1, p. 35-43, 2010.
SCHUBER, J. M.; MONTEIRO, L. B.; ALMEIDA, L. M.; ZAWADNEAK, M. A. C. Natural enemies associated to aphids in peach orchards in Araucária, Paraná, Brazil. Brazilian Journal of Biology, v. 72, p. 847-852, 2012. DOI: https://doi.org/10.1590/S151969842012000500010

SILVEIRA NETO, S.; MONTEIRO, R. C.; ZUCCHI, R. A.; MORAES, R. C. B. Uso da análise faunística de insetos na avaliação do impacto ambiental. Scientia Agricola, v. 52, n. 1, p. 9-15, 1995. DOI: https://doi.org/10.1590/S0103-90161995000100003

SILVEIRA NETO, S.; NAKANO, O.; BARBIN, D.; VILLA NOVA, N. A. (Eds.). Manual de ecologia dos insetos. São Paulo: Agronômica Ceres, 1976. 419p.

SOUZA, I. L.; MARUCCI, R. C.; SILVEIRA, L. C. P.; PAULO, N. C. P.; LEE, J. C. Effects of marigold on the behavior, survival and nutrient reserves of Aphidius platensis. Biocontrol, v. 1, p. 1-11, 2018. DOI: https://doi.org/10.1007/s10526-018-9882-8

SUJII, E. R.; PIRES, C. S. S.; VENZON, M.; FERNANDES, O. A. Controle de artrópodes-praga com insetos predadores. pp.113-140. In: FONTES, E. M. G. VALADARES-INGLIS, M. C. (Eds.). Controle biológico de pragas da agricultura. Brasília: Embrapa, 2020. 510p.

TIBOLA, C. S.; FACHINELLO, J. C.; GRÜTZMACHER, A. D.; PICOLOTTO, L.; KRUGER, L. Manejo de pragas e doenças na produção integrada e convencional de pêssegos. Revista Brasileira de Fruticultura, v. 27, p. 215-218, 2005. DOI: https:// doi.org/10.1590/S0100-29452005000200008

ZART, M.; BOTTON, M.; FERNANDES, O. Injúrias causadas por moscadasfrutas sulamericana em cultivares de videira. Bragantia, v. 70, p. 6471, 2011. DOI: https://doi.org/10.1590/ s0006-87052011000100011 